\title{
Benefits and Challenges of the Use of High-Z Plasma Facing Materials in Fusion Devices
}

\author{
R. Neu \\ Max-PIanck-Institut für Plasmaphysik, EURATOM Association, Boltzmannstr.2, 85748 Garching, \\ Germany
}

\begin{abstract}
.
The use of high-Z plasma facing components requires intensive research in all areas, i.e. in plasma wall-interaction, in the physics of the confined plasma, diagnostic, and in material development. Only a few present day divertor tokamaks - mainly Alcator C-Mod and ASDEX Upgrade - gained experience with the refractory metals molybdenum and tungsten, respectively. ASDEX Upgrade was stepwise converted from graphite to tungsten PFCs and in parallel a reduction of the deuterium retention by almost a factor of ten has been observed due to the strong suppression of $\mathrm{D}$ co-deposition with carbon. The deuterium retained in $\mathrm{W}$ is in line with laboratory results. In order to diagnose $\mathrm{W}$ sources and the $\mathrm{W}$ content in the main plasma adequate spectroscopic methods had to be developed. As expected from the sputtering threshold of Mo and W, negligible erosion by the thermal divertor background plasma is found in ASDEX Upgrade and Alcator C-Mod under low temperature divertor conditions. However, erosion by fast particles and intrinsic impurities, which additionally might be accelerated in rectified electrical fields observed during ion cyclotron frequency heating, plays an important role. The Mo and $\mathrm{W}$ concentrations in the plasma centre are strongly affected by plasma transport and variations up to a factor of 50 are observed for similar influxes. However, it could be demonstrated that sawteeth and turbulent transport driven by central heating can suppress central accumulation. The inward transport of high- $Z$ ions at the edge can be efficiently reduced by 'flushing' the pedestal region caused by frequent edge instabilities. Since with metal walls the edge radiation by low- $Z$ impurities is reduced, it has to be substituted in a pure high$\mathrm{Z}$ device by artificially injected low-Z impurities in order to keep the power load at an acceptable level. Experiments at ASDEX Upgrade suggest that a regime with benign erosion and favourable confinement can be achieved. Extrapolations to ITER and DEMO are difficult since the physics of plasma transport is not yet completely understood, the particle and energy fluxes are orders of magnitude higher and the technical boundary conditions in DEMO strongly differ from those of present day devices.
\end{abstract}

Keywords: Plasma-Material Interactions, Tungsten, Impurities in Plasmas, Fusion reactor materials, Tokamaks, Power Exhaust

PACS: $52.40 . \mathrm{Hf}, 52.25 . \mathrm{Vy}, 28.52 . \mathrm{Fa}, 52.55 . \mathrm{Rk}, 52.55 . \mathrm{Fa}$

\section{INTRODUCTION}

After the decision for the construction of ITER, plasma wall interaction moved strongly into the focus of magnetic confinement fusion research because it can sensitively influence the plasma performance and reactor availability. A future reactor cannot rely on low-Z plasma facing components (PFCs) due to the strong degradation of their thermomechanical properties under neutron irradiation and the high expected erosion. Moreover, there are strict limits on the tritium in-vessel inventory, not allowing large amounts of $\mathrm{T}$ co-deposited with carbon or beryllium. Therefore a solution with high-Z metal as armour material has to be developed, or a completely different approach as for example 
liquid PFCs has to be adopted (see for example [1]), which - however - lies outside the scope of this paper. Due to its highest melting temperature, very high sputtering threshold and its rather benign radioactive behaviour after neutron irradiation, tungsten $(W)$ is the first choice for the use at PFCs, although molybdenum (Mo) and its alloys can serve in most cases as an equivalent in the investigations in present day devices.

The use of high-Z PFCs requires intensive research in all areas, i.e. in plasma wallinteraction, in the physics of the confined plasma, diagnostic, and in material development. Nevertheless the fusion community only reluctantly uses them as plasma facing materials (PFMs). The reason can be found in its strong ability to hamper plasma operation as found in early limiter tokamak experiments and - on the other side - the very beneficial behaviour of carbon based PFCs in respect to power handling capabilities and plasma compatibility. In early days of fusion research the vacuum compatibility of the in-vessel components was one of the highest priorities. For example, the vacuum liner in the ORMAK tokamak was coated with gold, which was selected because its chemical inertness [2]. Therefore, it was a small step to the use of refractory metals as PFM when going to devices with higher heating capabilities and therefore higher demands for the power handling by the PFCs. However, by improving the vacuum and the conditioning of the vacuum vessel, which essentially means the reduction of oxygen and carbon and their compounds, the plasma properties also improved, but at the same time strong central radiation from the high- $Z$ material became evident. Eventually, this led to a degradation and even to hollow electron temperature profiles as observed in the PLT tokamak [3]. Following these observations the route for the PFC diverged into two branches of tokamak devices: High field tokamaks $\left(B_{t}>5-8 \mathrm{~T}\right)$ operating at high current and high plasma densities kept the high Z-components. Tokamaks operating at moderate current densities, i.e. devices with larger cross section exchanged their high- $Z$ components for medium- $Z$ materials (as stainless steel) and finally to low- $Z$ materials as graphite or even beryllium. Earlier reviews on the experiments and results with high- $Z$ plasma facing components can be found in $[4,5,6]$. Today, Alcator C-Mod (C-Mod) and ASDEX Upgrade (AUG), which belongs to the second branch but has progressively exchanged its graphite PFCs to W PFCs during the last ten years, are the only two divertor tokamaks using all refractory PFCs (Mo and $\mathrm{W}$, respectively). There are a two other tokamaks - TRIAM-1M [7] and FTU [8]- which use Mo limiters and JT-60U just recently equipped $1 / 21$ of the toroidal circumference of its outer divertor with $\mathrm{W}$ coated graphite tiles [9]. Additionally, the limiter tokamak TEXTOR [10, 11, 12] performs various experiments with Mo and $\mathrm{W}$ test-limiters, exploring mainly erosion and melting of both materials.

This paper presents a short overview on recent results related to the use of tungsten in fusion devices. The second section describes the development of the spectroscopic diagnostic of $\mathrm{W}$ which has been performed in Tokamaks as well as in plasma simulators and electron beam ion traps (EBITs). The following sections will summarize the present knowledge on the hydrogen retention in $\mathrm{W}$ and Mo, the effect of high particle fluences, the $\mathrm{W}$ erosion and the $\mathrm{W}$ transport into and within the main plasma. Finally, before the conclusion, an extrapolation to ITER is attempted and the main issues for DEMO on top of it are sketched. 


\section{DIAGNOSTIC OF W IN FUSION DEVICES}

Emissions from neutral tungsten and from tungsten ions with low charge number allow to quantify the $\mathrm{W}$ influx. In the main plasma, spectral lines from highly charged ions can be used to derive the local $\mathrm{W}$ density. Large progress on the tungsten spectroscopy in fusion devices has been made since the early investigations in tokamaks. Equally, the production and benchmarking of atomic data for the interpretation of the measured spectra has been considerably enforced. Under the premises of using spectroscopy as a quantitative tool, the excitation rates and in the case of non-resonant transitions, even the setup of a collisional radiative model is necessary. However, not only the rates within one ionisation stage need a careful revisiting, but also the ionisation and recombination rates as could be extracted from tokamak measurements and in EBIT investigations on the ionisation equilibrium.

Since recombination can be neglected at the edge of low density fusion plasmas, the influx $\Gamma_{i n}$ can be approximately determined from the photon flux $\Gamma_{p h}$ through the inverse photon efficiency $S / X B$ without detailed knowledge of the local electron density $\left(\Gamma_{i n}=\right.$ $\frac{S}{X B} \Gamma_{p h}$ ), where $B$ is branching ratio, $S$ the ionisation rate coefficient of the observed ionisation stage and $X$ the excitation rate coefficient to the upper level of the transition, both of which are temperature dependent [13]. For transitions with energies close to the ionisation energy, $S / X B$ is only weekly dependent on the electron temperature and under typical conditions (influx of particles against a temperature gradient) $T_{e} \approx 1 / 3 E_{\text {ion }}$, with $E_{i o n}$ denoting the ionisation potential is a good estimate. In practice, $S$ and $X$ are often calculated effective rates taking into account also the density dependence. The spectroscopic measurement has to be performed in the direction of the impurity influx and for a laterally non-isotropic particle source the whole emission cloud has to be observed. A recent review on the spectroscopic diagnostic of tokamak edge plasmas is given in [14]. In the case of $\mathrm{W}$, there exists only a small experimental dataset from the former PSI-1 plasma generator device (Berlin) for a WI transition $\left(5 d^{5} 6 s 7 S_{3}\right.$ $\left.5 d^{5} 6 p^{7} P_{4}\right)$ at $400.88 \mathrm{~nm}$ [15] which was verified at ASDEX Upgrade under fusion plasma conditions [16] and from recent investigations at the tokamak TEXTOR [17] on a few other suitable spectral lines in the visible and UV spectral range.

The investigation on $\mathrm{W}$ emissions from the central plasma are limited to charge stages below $q \approx 50+$ in present day fusion devices. The most intense spectral features of $\mathrm{W}$ for these plasma conditions are observed between 0.4 and $0.8 \mathrm{~nm}$, around $5 \mathrm{~nm}$ and between 10 and $30 \mathrm{~nm}$ (see $[18,19]$ and references therein). The lines at $0.4-0.8 \mathrm{~nm}$, are emitted by ionisation stages from Br-like $\mathrm{W}^{39+}$ to about Mn-like $\mathrm{W}^{49+}$. Investigations in EBITs have confirmed the identification of the spectral lines, moreover - in some cases - they provided the only clue to unambiguously identify the transitions [20, 21, 22]. The modelling in that wavelength range agrees well for the distribution of emissions versus the wavelength. The intensities of single spectral lines are predicted typically with deviations of around factor 2, while the wavelengths for transitions in the soft Xray spectral range are predicted within $0.002 \mathrm{~nm}$.

The experience gained in this recent experiments and atomic physics calculations encourage the use of this tools to extrapolate from the current experimental findings to spectra as they might be observed in future experiments. However, the extrapolation 


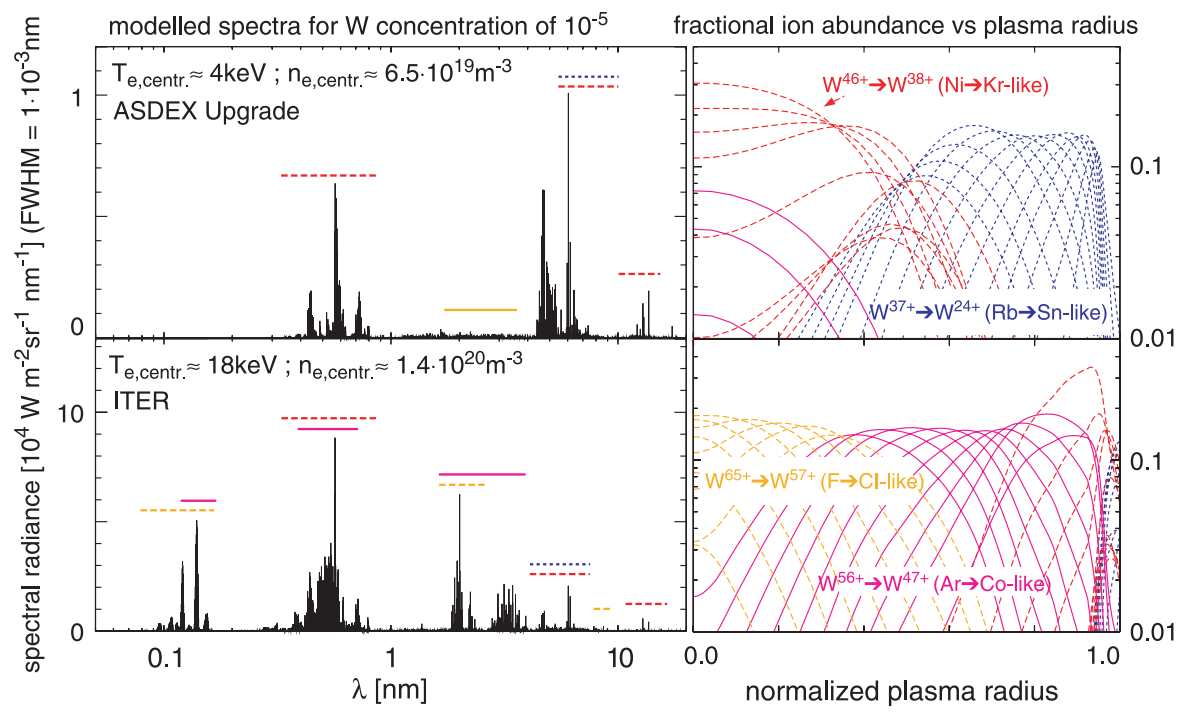

FIGURE 1. Left: modelled spectra for a typical ASDEX Upgrade discharge $\left(T_{e, c e n t r a l} \approx 4.0 \mathrm{keV}\right)$ and a projected ITER discharge $\left(T_{e, \text { central }} \approx 18 \mathrm{keV}\right)$ for a central W concentration of $10^{-5}$. Right: the underlying ionisation balance versus normalised plasma radius, taking estimations for impurity transport into account (Figure taken from [19]).

in electron temperature / ionisation stages is quite far and currently no fusion device is at hand to bridge this gap. Calculations for the ITER standard scenario, where a central electron temperature $\mathrm{T}_{e} \approx 18 \mathrm{keV}$ is envisaged, reveal that the above described emissions will occur at the outer half of the plasma. New spectral features will be emitted from mid-radius to the plasma centre by ionisation stages of Cr-like $\mathrm{W}^{50+}$ to $\mathrm{C}$-like $\mathrm{W}^{68+}$. Important spectral ranges will be at $0.1-0.15 \mathrm{~nm}$ and $1.8-3.5 \mathrm{~nm}$ for ionisation stages of Co-like $\mathrm{W}^{47+}$ to $\mathrm{C}$-like $\mathrm{W}^{68+}$. Predictions of spectral lines around $7-8 \mathrm{~nm}$ for the ionisation stages above $\mathrm{Cl}$-like $\mathrm{W}^{57+}$ are relatively weak, but seem also to be an interesting alternative in the VUV spectral range. Figure 1 shows simulated spectra for typical plasma parameters measured in ASDEX Upgrade and predicted for ITER. In very recent EBIT investigations [23, 24, 25] most of the predicted lines could be identified. However, due to the different electron densities in the EBIT $\left(n_{e} \leq 10^{19} \mathrm{~m}^{-3}\right)$ and in the centre of fusion plasmas $\left(n_{e} \geq 10^{20} \mathrm{~m}^{-3}\right)$ and the mono-energetic electron beam (EBIT) instead of a Maxwellian distribution, investigations in fusion devices are still indispensable, to explore the composition of the spectra emitted from fusion plasmas.

\section{HYDROGEN RETENTION}

As stated in the introduction, one of the major goals of replacing carbon based PFCs to tungsten PFCs is the reduction of the hydrogen retention. In parallel with the transition of AUG from graphite to tungsten, a reduction of the deuterium retention by almost a factor of ten has been observed. This is due to the strong suppression of D co-deposition with carbon as investigated by post-mortem surface analysis [26, 27]. Additionally, 


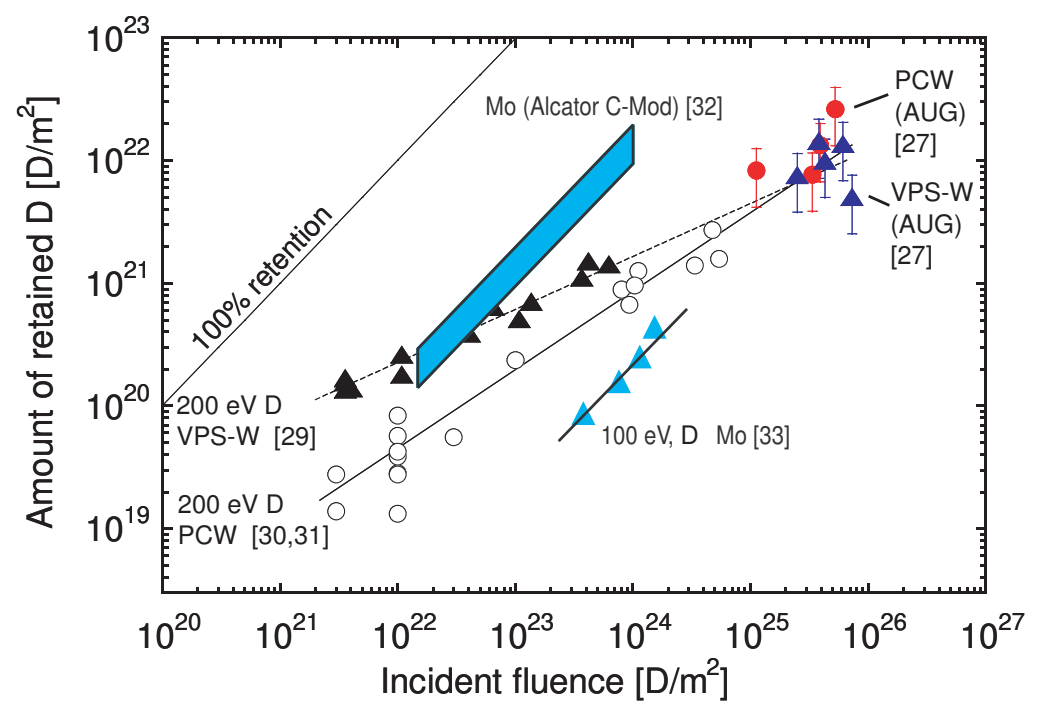

FIGURE 2. Deuterium retention in $\mathrm{W}$ and Mo. The data are taken from the references indicated in the figure. The laboratory measurements are performed at room temperature. Please note, W (Poly Crystalline (PC) and Vacuum Plasma Spray (VPS)) was irradiated with $200 \mathrm{eV}$ deuterium and Mo was irradiated with $100 \mathrm{eV}$ D. Whereas laboratory data and data taken from AUG fit together, there is a discrepancy by more than a factor of 10 in the case of Mo in Alcator C-Mod.

dynamic gas balance measurements, where the retention is derived from the difference of the puffed and the pumped amount of gas [28] show a similar reduction, in line with expectations from laboratory measurements on the deuterium retention in $\mathrm{W}[29,30$, 31].

In Alcator C-Mod the hydrogen retention was studied using a 'static' gas balance method, where all pumps are switched off or separated from the vacuum vessel and the retained amount is calculated from the injected gas puff and from the pressure inside the vacuum level after the discharge [32]. The Mo surfaces are found to retain large fractions, $20-50 \%$ of the $\mathrm{D}_{2}$ gas fueled. It is concluded that the retention occurs through ion bombardment, implantation and diffusion to trap sites. The above number can also interpreted that roughly $1 \% \mathrm{D}$ of the incident ion fluence is retained. No saturation of the retention rate was observed even after $25 \mathrm{~s}$ of integrated plasma exposure, which is concluded to be consistent with trapping sites in Mo. Differences between C-Mod and laboratory retention results [33] are thought to be due to such factors as the multiply ionized low-Z ions incident on the surface directly creating traps, the condition of Mo (impurities, annealing) and the high-flux densities in the C-Mod divertor, which are 10 - 100 times those used in laboratory studies. These results are summarized in Fig. 2 together with results from laboratory measurements (see references given in the figure).

\section{Blisters, Bubbles and Mixed Material Effects}

Although the exposure of PFMs in present day tokamaks is the most realistic test of their behaviour under fusion plasma conditions, there is a big gap towards ITER or a 
reactor concerning the particle fluence impinging on the surfaces, because of the very low duty cycle of present day fusion devices. In order to close this gap, experiments are performed in (linear) plasma devices, allowing much larger irradiation times.

Under high hydrogen fluence, low surface temperature conditions $(<600 \mathrm{~K})$ blistering of $\mathrm{W}$ can be observed (see for example [34, 35]), which could increase the hydrogen inventory and lead to increased erosion if the blister cap is removed in course of power loading [36]. Many investigations have been performed to uncover the detailed conditions for the formation of blisters. Very recently, experiments performed in PISCES-B revealed [37] that blisters are completely suppressed and the hydrogen retention is drastically reduced, when $\mathrm{He}$ is added to the hydrogen in a percentage fraction.

In the temperature range of 1000 to $2000 \mathrm{~K}$ a nano-structure is formed at the $\mathrm{W}$ surface if it is exposed to $\mathrm{He}$ ions with energies above $20 \mathrm{eV}[38,39,40]$. Its formation is observed in pure He plasmas as well as in mixtures of He and $\mathrm{H}$. The thickness of this nano-structure can reach several microns, depending on He flux and fluence. The time dependence hints to a diffusional process that is slower than the diffusion of $\mathrm{He}$ in $\mathrm{W}$ but much faster than void/bubble diffusion [39], but other formation processes as for example the coalescence of helium bubbles at the surface are also discussed [40]. The impact of $\mathrm{W}$ surface nano-structure morphology on fusion reactor performance is not yet fully clear but these structures could potentially lead to a larger $\mathrm{W}$ erosion and to an increased dust production. It has to be noted, however, that the production of the nano-stucture is not yet observed in a fusion device and that all observations are in line with the fact that it develops only under conditions with negligible erosion.

If tungsten is not the only PFM in a tokamak, mixed material effects could influence the performance of the W PFCs. An especially obvious example for such an effect is the formation of a low melting Be-W alloy as observed in PISCES-B [41]. The formation of the alloy depends delicately on the amount of $\mathrm{Be}$ in the plasma, the plasma conditions leading to either deposition of $\mathrm{Be}$ or a steady erosion of $\mathrm{W}$ and the surface temperature which strongly influences the Be diffusion into $\mathrm{W}$ and its sublimation [42, 43, 44]. The complexity of this process requires detailed modelling (or experiments) in order to judge the potential impact of it.

\section{W EROSION AND MELTING}

Physical sputtering results from elastic energy transfer from incident particles to target atoms. Surface atoms can be ejected, if enough energy is transferred to overcome the surface binding energy. At low ion energies, where the transferred energy to surface atoms is comparable with the surface binding energy, the sputtering yield decreases strongly and becomes zero below a threshold energy. The threshold energy for the onset of sputtering from light projectiles on a substrate consisting of heavier species can be determined from momentum and energy conservation in an elastic collision. The sputtering thresholds for $\mathrm{H}, \mathrm{D}$ and $\mathrm{T}$ on $\mathrm{W}$ are $\approx 450 \mathrm{eV}, 210 \mathrm{eV}$ and $140 \mathrm{eV}$, respectively [45] and therefore the erosion by background ions is almost negligible and the observed erosion is mainly due to intrinsic impurity ions due to their higher mass and electrical charge. In C-Mod, a good agreement between measured and simulated influx could by achieved for ohmic discharges when taking Mo self-sputtering and sputtering by $\approx 2 \%$ 


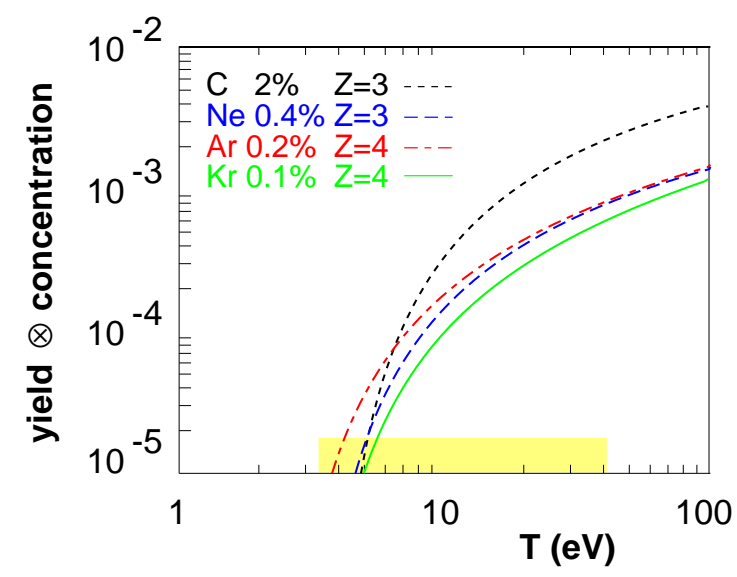

FIGURE 3. Tungsten sputtering yields for different species versus plasma temperature, assuming $E_{\text {impact }}=3 Z T+2 T$. Total yields are multiplied by the assumed concentrations to obtain realistic, effective yields per fuel ion flux. Charge states used for the calculation are given in the inset.

$\mathrm{B}^{3+}$ (from boronisation) into account [46]. Similarly, W sputtering in ASDEX Upgrade could be explained in a wide range of divertor plasma temperatures by assuming an admixture of 1-2\% low-Z impurities $(\mathrm{C}, \mathrm{O})$ in the charge state of $Z=4$ [15]. An obvious question is whether impurity seeding leads to a higher $\mathrm{W}$ source due to more efficient sputtering by heavier species impinging at a potentially higher charge state resulting in higher energies. Experiments with $\mathrm{Ne}$ and $\mathrm{N}_{2}$ seeding in ASDEX Upgrade $[15,47]$ showed that at least inbetween ELMs (see below) a reduction of the $\mathrm{W}$ influx could be achieved due to the strong cooling of the divertor plasma which overcompensates the higher sputtering yield of the impurity ions. Beside this beneficial effect on divertor power load and $\mathrm{W}$ erosion an improved confinement has been observed recently in ASDEX Upgrade when using nitrogen as a seeding gas [48]. In general, similar erosion yields (using the yields from [49]) are expected for ITER relevant divertor parameters using estimated concentrations and charge states relevant to ITER. As can be judged from Fig. 3, there is virtually no difference in $\mathrm{W}$ sputtering rates for $\mathrm{Ne}, \mathrm{Ar}$ and $\mathrm{Kr}$ at their corresponding concentration in the range of typical SOL and divertor temperatures.

When comparing the gross erosion, typically measured by spectroscopical means, with the net erosion from probe measurements, a difference by a factor of up to 10 is found under low temperature high density conditions [15]. This is attributed to 'prompt redeposition' as it was already observed in W marker experiments [50]: For high-Z materials the gyro-radius in the external field can be larger than the ionisation length, which can lead to deposition of the eroded particle directly after its erosion. More recent investigations show about a factor of three reduction for campaign integrated erosion in the divertor of ASDEX Upgrade [51], which may be attributed to the fact that the divertor plasma is not always dense enough for a high prompt re-deposition fraction. Details of this process are still under investigation, because it could diminish further the low sputtering yield under ITER high density, low temperature conditions.

Besides the erosion by a thermal steady state plasma, erosion by transients can play an important role, because they can lead to increased yields or even to melting of the 


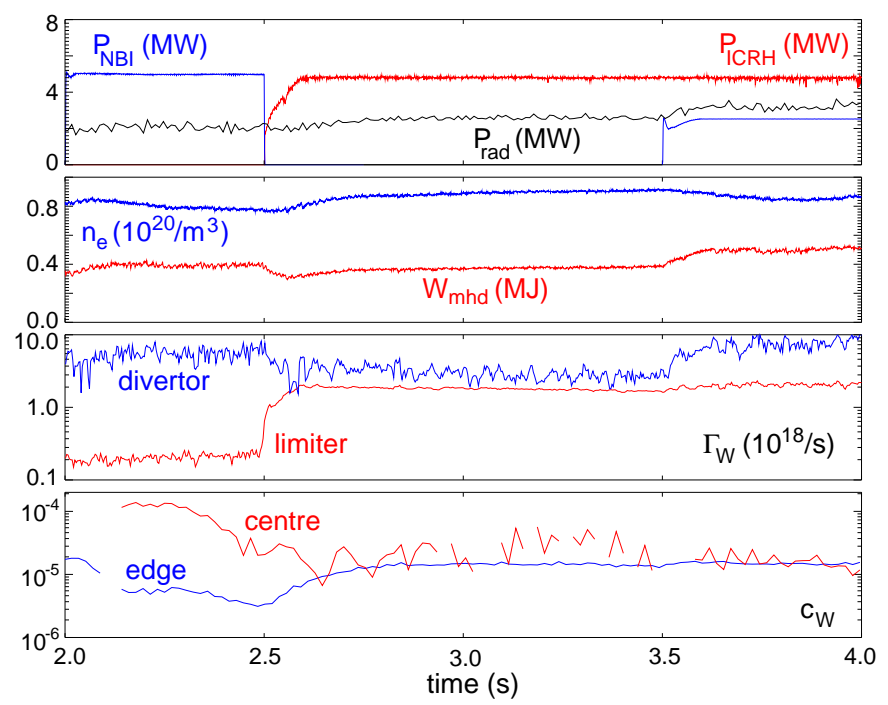

FIGURE 4. $W$ influx and $\mathrm{W}$ concentration in ASDEX Upgrade during discharge \#23476. The two top graphs present the auxiliary heating power $\left(P_{N B I}, P_{I C R H}\right)$ and total radiation $\left(P_{\text {rad }}\right)$ as well as the line averaged density $\left(n_{e}\right)$ and the stored energy $\left(W_{m h d}\right)$ of the plasma. The third graph highlights the $\mathrm{W}$ influx $\left(\Gamma_{W}\right)$ from the limiters and the divertor and the bottom insert shows the deduced $\mathrm{W}$ concentration $\left(c_{W}\right)$ at the plasma edge and the centre (Figure from [55]).

surfaces by the rapid energy deposition. In H-Mode (high confinement mode) plasmas, which are envisaged as the standard operating scenario for ITER, there are periodic edge instabilities observed, ejecting particles and energy on a sub ms timescale. During these so called edge localized modes (ELMs), not only the particle flux is increased, but also the particles' energy, because they originate from the hot edge region ('pedestal') of the main plasma. Measurements in ASDEX Upgrade using high spatial and temporal resolution reveal, that under high density conditions, the W sputtering in between ELMs is strongly suppressed and that the sputtering during ELMs contributes up to $90 \%$ of the total W erosion [51]. Another important process for increasing the sputter source of high-Z PFCs is the acceleration of plasma and impurity ions in the rectified sheath due to ion cyclotron resonance heating (ICRH). This is reported from Alcator C-Mod as well as from ASDEX Upgrade [52, 53, 54, 51], where the Mo fluxes and W fluxes, respectively, increase by about a factor of ten during the operation of ICRH. In both experiments it is also found that the divertor impurity source is almost unchanged and, although being still larger than the limiter source, the impurity concentration in the main plasma is clearly dominated by the limiter source. This hints to a good divertor retention of Mo and W, respectively, which was also determined quantitatively by trace $\mathrm{W}$ injections in ASDEX Upgrade [16]. As an example, Figure 4 shows the temporal evolution of some plasma parameters for an ASDEX Upgrade discharge (\#23476) with different heating methods [55]. The W-influx is deduced from the W I line at $400.9 \mathrm{~nm}$ as described in [51]. The W concentration is deduced from the quasicontinuum emission at $5 \mathrm{~nm}$ and the spectral line at $0.794 \mathrm{~nm}$ emitted from Ni-like $\mathrm{W}^{46+}$ [19]. The first one gives the $\mathrm{W}$ concentration close to the plasma edge (around $T_{e} \approx 1 \mathrm{keV}$ ), whereas the latter one represents the central concentration $\left(T_{e} \approx 3 \mathrm{keV}\right)$ in typical ASDEX Upgrade discharges. During the 
first phase (until $t=2.5 \mathrm{~s}$ ) the plasma is heated by neutral beam injection (NBI) only. During this phase the $\mathrm{W}$ source at the limiter in the main chamber is more than a factor of ten smaller than the divertor $\mathrm{W}$ source. At the same time the $\mathrm{W}$ concentration is strongly peaked, as can be seen from the ratio of the central to edge $\mathrm{W}$ concentration, which is about 20 . This strong peaking is usually explained by neoclassical inward drifts (see next section), which can be dominant in the absence of large turbulent transport or macroscopic instabilities as sawteeth [56]. At $t=2.5 \mathrm{~s}$ the NBI is switched off and at the same time the ion cyclotron resonance heating (ICRH) is switched on. Immediately, the limiter source increases by a factor of 10 . On a longer timescale - reflecting the 'slow' particle transport within the plasma - the edge $\mathrm{W}$ concentration rises (by about a factor of 4), but at the same time the central concentration decreases to a value close to that at the edge. This phase is characterised by dominant anomalous transport, which tends to reduce $\mathrm{W}$ density gradients. In the third phase of the discharge from $t=3.5 \mathrm{~s}$ on, a part of the NBI is switched on again, resulting in an increased W source in the divertor, which is not reflected in the main chamber concentrations at all, consistent with the above mentioned behaviour.

As an additional erosion process, $\mathrm{W}$ erosion by arcs was investigated post mortem in AUG [57]. The arc tracks were observed at different locations, i.e. at tiles with direct plasma contact, such as divertor targets, as well as components remote from the plasma. The track orientation and pattern allow to distinguish between arcs burning during glow discharges and during plasma operation. The arcs during plasma operation clearly dominate and are especially found at the baffle region of the inner divertor in a $10 \mathrm{~mm}$ wide region near to the leading edge of the individual tiles around the whole toroidal circumference. They are covering more than $10 \%$ of this area and removed the complete tungsten coating $(3-4 \mu \mathrm{m})$. Droplets splashed from the arc track are detected at the surface close to the arc. Similar droplets are found all over the vessel [58], pointing to the fact that arcs could significantly contribute to the dust inventory. This is especially important since this source can barely be accounted for by spectroscopic means and estimations on dust productions based on spectroscopic erosion measurements could significantly underestimate the actual value.

As stated already above, a further erosion mechanism could be melting and subsequent melt layer losses. However, in ASDEX Upgrade, there is not enough energy deposited neither during ELMs nor during disruption, to cause melting. Although surface melting is observed during disruptions in Alcator C-Mod, a quantitative assessment is not available yet. Controlled experiments on melt layer behavior are mainly performed in TEXTOR using bulk W test limiters [59]. Spectroscopic measurements of the atomic tungsten flux from a hot $\mathrm{W}$ surface indicate that no enhancement of atomic tungsten release exceeding physical sputtering and normal thermal sublimation for temperatures below $3700 \mathrm{~K}$ occurs under the experimental conditions in a limiter Tokamak. The experiments with different types of tungsten limiters in TEXTOR also demonstrate that liquid tungsten can move rapidly. The motion is perpendicular to magnetic field lines and it was attributed to the thermo-electron emission current and the resulting $j \times B$ force. As a result, tungsten melting can lead to a large material redistribution without ejection of molten material to the plasma. 


\section{TRANSPORT AND SUPPRESSION OF CENTRAL ACCUMULATION}

Apart from the influx, the central impurity concentration depends strongly on transport which can be divided in the region of open field lines, the so called scrape off layer (SOL) and the confined plasma. The experimental investigations on transport in the SOL region are scarce and rather indirect. By use of a sublimation probe, $\mathrm{W}$ was injected in the divertor and at the midplane SOL of ASDEX Upgrade [16], revealing a divertor retention of 16 in a medium density $\mathrm{H}$-mode discharge in line with the observation described in the previous section and results from C-Mod [52]. In H-modes, one has to subdivide the confined plasma into the pedestal and core region. In the reactor relevant regime of typeI ELMs, the pedestal, with its steep pressure gradient, breaks down during an ELM and a substantial part of the pedestal plasma is ejected. In between ELMs, tungsten moves into the pedestal region due to strong inward particle drift [60, 61]. If the next ELM arrives in due time this tungsten is removed, before further penetrating towards the central plasma. The beneficial effect of frequent ELMs on the reduction of the W confinement time has been studied in detail in [62]. An increase of the ELM frequency and a reduction of the $\mathrm{W}$ content can also be achieved by external means, either by an increased gas puff rate (see also Fig. 5) or by so called ELM pace making [63, 64, 65].

In the core plasma, an inward particle drift can lead to accumulation in the centre.
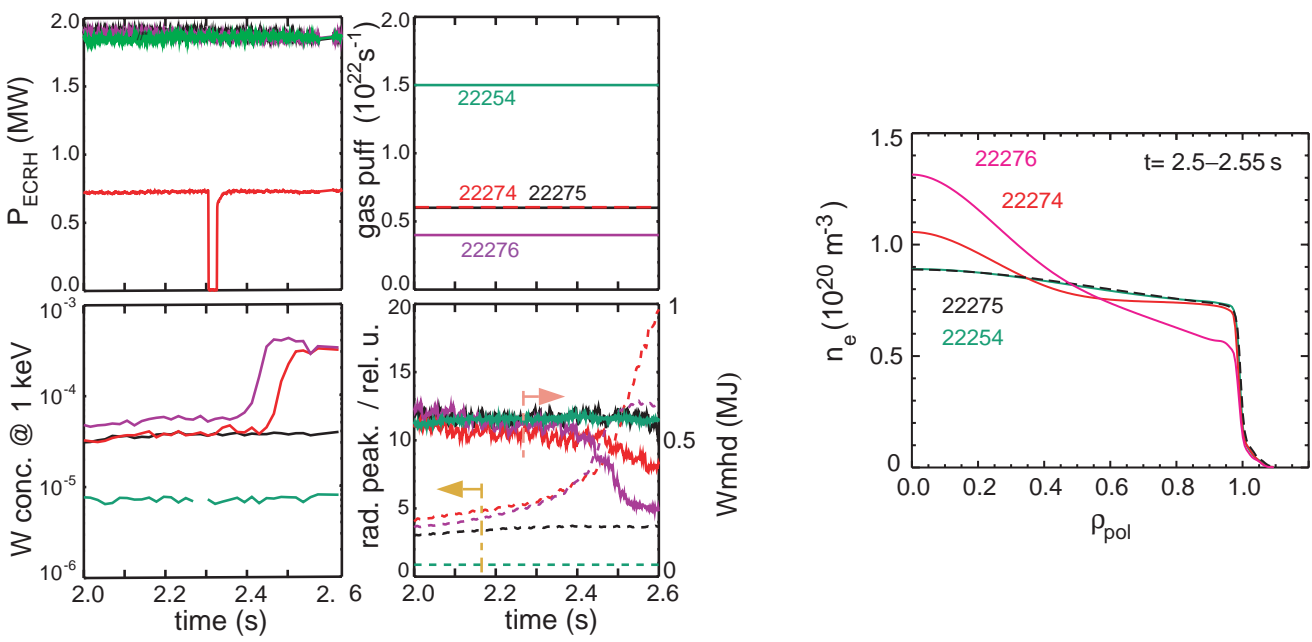

FIGURE 5. Time traces and density profiles of four discharges with different levels of central ECRH heating and gas puff. Cases with too low gas puff or ECRH power exhibit density peaking and central $\mathrm{W}$ accumulation $\left(P_{N B I}=5 \mathrm{MW}, I_{p}=1 \mathrm{MA}(\operatorname{after}[47])\right)$.

In a simple picture, the diffusion consists of an anomalous and a neo-classical part $D=D_{a n}+D_{\text {neo }}$. Recently it has been shown that $D_{a n}$ is only weakly $Z$ dependent and that the anomalous convective part is usually very small for higher $Z$ [66]. Therefore the convective contribution is assumed to be purely neo-classical with $v=v_{\text {neo }}$. If the deuterium density profile is not particularly flat this, in general, leads to accumulation of high- $Z$ elements in the core. The neoclassical accumulation has been experimentally observed in several devices $[67,65,68,69,70]$. However, if the heat flow in the core is sufficiently high, anomalous transport can easily exceed the neoclassical effects, espe- 
cially that of high-Z ions because $D_{\text {neo }}$ decreases with $Z$. Therefore increasing $D_{a n}$ has a much stronger effect on the impurity density profile, than on the background plasma. Consecutively a small increase of $D_{a n}$ deteriorates the performance only weakly while suppressing strongly the high- $Z$ contamination. First hints for a beneficial influence of central ICRH on the central radiation where already seen in $\mathrm{W}$ test-limiter experiments in TEXTOR [71]. However, the effect of central heating reducing central impurity accumulation by stimulating anomalous transport was first identified in ASDEX Upgrade [72] and the recipe was confirmed in several other devices [70, 73, 9]. The effect is clearly seen in Fig. 4 and is further exemplified in Fig. 5, which demonstrates the strong correlation of the $\mathrm{W}$ accumulation with the background density peaking at ASDEX Upgrade. In the discharges presented in Fig. 5, the central ECRH heating power and gas flux were varied. Two discharges with sufficient central ECRH and gas puff are stable, while the discharges with the lowest gas puff and the lowest ECRH power showed central density peaking in combination with $\mathrm{W}$ accumulation. A runaway situation is obtained when the tungsten radiation in the plasma centre approaches a considerable fraction of the local heating power flux. It is is suppressed by increasing the gas puff, which reduces the $\mathrm{W}$ source and increases the edge transport keeping the edge $\mathrm{W}$ concentration and by the central ECRH which acts on the W accumulation.

\section{EXTRAPOLATION TO FUTURE DEVICES}

One of the main arguments for ITER to start with a CFC divertor, is the huge power deposition during unmitigated transients, which are expected to appear in the initial operational phase [74] and which could easily lead to melting of W components [76, 75]. Since present day tokamaks or barely capable of reaching such conditions, it is not clear how these melt layer will evolve and what consequences have to be expected for the lifetime of the components and for the contamination of the main plasma. Although extensive modeling has been performed to address the questions raised above (see for example [77] and references therein), it is not clear yet how large the melt layer losses will be and whether vapour shielding can efficiently lower the melt erosion. Recent simulations based on experiments performed in plasma guns and quasi-stationary plasma accelerators predict a strong distortion of the surface already after 100 ELMs with an energy of $1.6 \mathrm{MJ} \mathrm{m}^{-2}$ and a duration of $\tau=0.5 \mathrm{~ms}$ [78]. In [80] and [79] two complementary approaches were made to extrapolate the deuterium retention data from present day tokamaks and laboratory devices to the tritium retention in ITER. Different scenarios concerning the material mix for the first wall and divertor were investigated (see Figure 6). Both investigations came to the conclusion, that the T-retention is mainly governed by co-deposition when using the low-Z PFCs Be and C. According to this analyses the initial ITER PFM mix will lead to a much larger T inventory (more than a factor of 10) compared to a full $\mathrm{W}$ device, where the $\mathrm{T}$ inventory is governed by implantation and retention in traps. It has to be stated that there is only very little information on the role of $n$-induced traps, which could increase the amount of retained $\mathrm{T}$ when approaching 1 dpa, the amount of neutron damage expected during the lifetime of the ITER divertor. However, only very recently data were presented, which were gained in experiments using $\mathrm{Si}$ ions to simulate the $\mathrm{n}$-damage, pointing to a very small increase of $\mathrm{T}$ retention by 


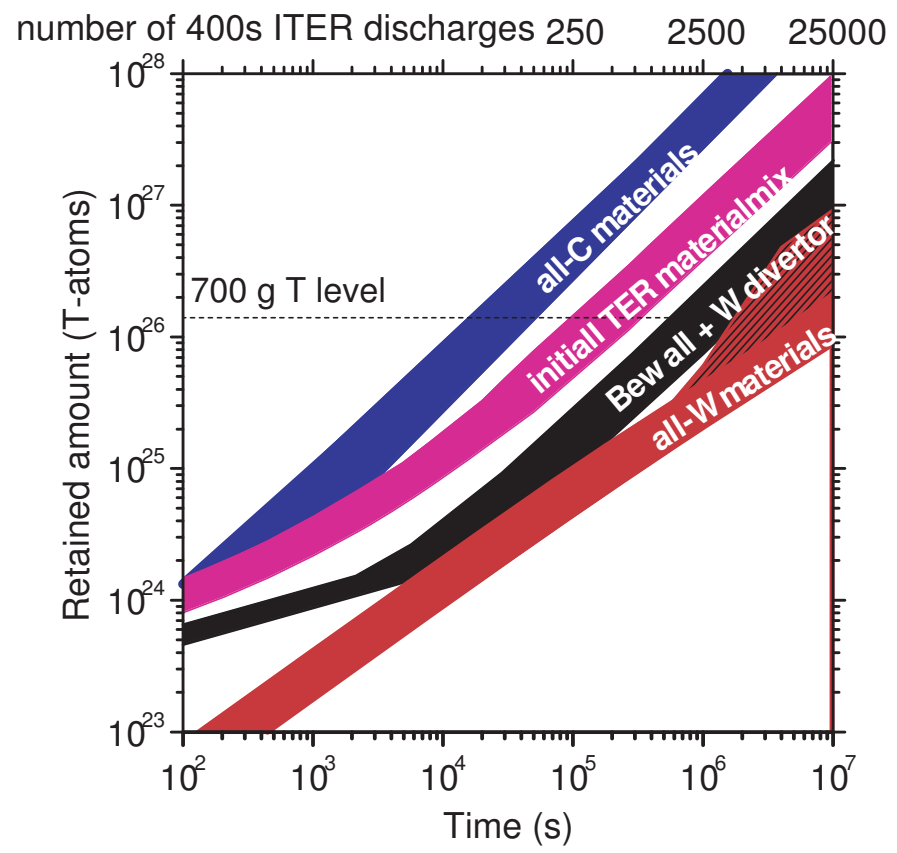

FIGURE 6. Tritium inventory in ITER for the all-C (blue line) and all-W options (red line) compared to the initial material choice $\mathrm{CFC} / \mathrm{W} / \mathrm{Be}$ (magenta). The black band indicates retention values for the option of a full-W divertor and Be first wall. The width of the bands indicates error margins essentially given by uncertainties in the incident wall fluxes. For $\mathrm{W}$ also an estimated contribution due to n-damage is introduced (hatched area)(after [79]).

n-induced traps under ITER conditions [81].

Due to the very narrow temperature range where alloying of Be with $\mathrm{W}$ takes place $(900$ to $1500 \mathrm{~K})$, no alloying is expected under steady state conditions in ITER [42, 43, 44] and even the deposited Be may actually alleviate potential problems caused by $\mathrm{W}$ blistering and the formation of nanoscopic W morphology due to the He irradiation [82]. However, since excursions to higher temperatures will be induced by transients, the appearance of alloying or the change in surface morphology, which would result in larger erosion rates and dust production, cannot be completely excluded and calls for further investigations.

Concerning the plasma transport of W, DIVIMP calculations based on B2-Eirene simulations suggest that even for a full $\mathrm{W}$ coverage of the ITER PFCs edge concentrations below $2 \cdot 10^{-5}$ can be expected for high density operation [83]. These calculations use 'averaged' transport coefficient scaled from edge parameters in present day devices. However, as observed in ASDEX Upgrade, there is a delicate balance of the mostly inward directed impurity transport in between ELMs and the 'flushing' of the impurities during the ELM. Whether this effect is approximated adequately with the 'averaged' transport of the simulations is not clear yet. The need for frequent ELMs as a necessary prerequisite for sufficiently low tungsten content in the plasma goes in line with the demand of ITER to keep the energy per ELM small. However, it has to be stated, that the scaling of the 'flushing' effect is not yet investigated. Suppression of ELMs by increasing the overall edge transport with edge resonant magnetic field perturbations 
as pioneered by DIII-D [84, 85] and JET [86, 87], may also be a solution. However, common to these experiments is the tendency that the separatrix temperature increases during the ELM suppression phases, which is consistent with an increase of $Z_{\text {eff }}$ compared to similar discharges without edge perturbation as reported by [88] and which would be counter productive in the case of high-Z PFCs.

Simulations for the central transport in ITER with the ASTRA code using GLF23 model [89] suggest that the anomalous particle transport should be significantly larger than the neoclassical one, leading only to very moderate peaking of the $\mathrm{W}$ concentration $[90,65]$. Moreover, recent theoretical work $[91,66]$ shows two dominant turbulent transport mechanisms for high Z-ions. Neither of them leads to a substantial accumulation of tungsten with respect to the deuterium density.

The extrapolation to reactor is even more difficult than to ITER, since the boundary conditions are completely different to present day devices. The main differences are the much higher operating temperatures (coolant at $600-700^{\circ} \mathrm{C}$ ) and the huge neutron fluence $\left(\approx 10 \mathrm{MWa}^{-1} \mathrm{~m}^{-2}\right.$ at the end of lifetime [92]), to which the components will be exposed. The high temperature is needed to yield a high thermodynamical efficiency. It will strongly reduce the $T$ retention and may anneal the defects caused by neutron irradiation, but it will also strongly promote the $\mathrm{T}$ penetration through the components leading to a new class of challenges. The combination of the high power load and the high temperature of the cooling medium will allow almost no transients, requesting operation modes not achieved so far. A much more elaborate analysis on the challenges for plasma facing components of a future fusion reactor is presented in [93].

\section{CONCLUSION}

The use of tungsten PFCs requires intensive research in all areas, i.e. in plasma wallinteraction, in the physics of the confined plasma, diagnostic, and in material development. Only a few present day divertor tokamaks - mainly Alcator C-Mod and ASDEX Upgrade (AUG) - gained experience with the refractory metals molybdenum and tungsten, respectively.

In ITER and future fusion reactors the measurement of the central $\mathrm{W}$ concentration will be of utmost importance because its potentially deleterious effect on the fusion power production due to its high cooling factor, resulting from the fact that it is only partly ionized in the confined plasma, even at reactor relevant temperatures in the range of 10 to $20 \mathrm{keV}$. The resulting strong radiation thus sets an upper limit for an acceptable $\mathrm{W}$ concentration of only a few $10^{-5}$. In this context, W spectroscopy will be one of the key diagnostics to optimise fusion performance by providing input for control tools to minimise the $\mathrm{W}$ source and the $\mathrm{W}$ density in the plasma. Although a quite large hydrogen retention of Mo is found in Alcator C-Mod, the reason for which is not yet resolved, the expected strong reduction of hydrogen retention is observed in ASDEX Upgrade after the transition to all W PFCs, in line with results from plasma simulators and laboratory experiments. The sputtering threshold of Mo and $\mathrm{W}$ is quite high, such that in contrast to carbon PFCs, negligible erosion by thermal divertor background plasma is found in these experiments. In contrast, erosion by fast particles and intrinsic impurities, which additionally might be accelerated in rectified electrical fields observed during ion cy- 
clotron frequency heating, plays an important role. The $\mathrm{W}$ concentration in the plasma centre is strongly affected by plasma transport and variations up to a factor of 50 are observed for similar influxes, due to a pronounced neoclassical inward drift in the main plasma as well as in the pedestal region of H-modes. However, it could be demonstrated that sawteeth and turbulent transport driven by central heating can suppress central W accumulation. The inward transport of high- $Z$ ions at the edge can be efficiently reduced by 'flushing' the pedestal region provided by frequent edge instabilities (ELMs). Among the challenges remain the strong increase of the $\mathrm{W}$ source and concentration resulting from ICRH and the need for rigorous modelling for the extrapolation of the results from present day devices to ITER. Most of them are currently addressed and results are expected in the upcoming years. Clearly, not all questions posed by ITER can be answered in present high- $Z$ devices, amongst which are the effects of material mixing with $\mathrm{Be}$, the melt behaviour under transients or the change of the hydrogen retention due to damage by high energy neutron irradiation [79]. Some answers may be provided by the ITER-like wall project in JET [94], which will employ a similar configuration of PFCs as ITER, namely Be in the main chamber and tungsten in the divertor, but others have to be answered by dedicated experiments in other plasma devices or by modelling. Similar conclusions are drawn in a very recent paper by Brooks et al. [95], in which a very beneficial behaviour is predicted for ITER equipped with all W PFCs under the condition that the effects of ELMs can be mitigated. Looking further into the future to a DEMO device there will be a new class of challenges, which will even not be tackled sufficiently in ITER. The main differences will be the necessity to operate the PFCs at high temperature and the neutron fluence they receive during their lifetime. From these two issues there result a lot of consequences not only for the PFCs themselves but also for the plasma scenarios. Therefore, developing PFCs for DEMO and beyond will require a focused R\&D effort on high heat flux components, but also large advances in the control of the edge plasma especially the complete suppression of large transients.

\section{ACKNOWLEDGMENTS}

The author would like to thank the teams of Alcator C-Mod, ASDEX Upgrade, JT-60U, PISCES-B and TEXTOR as well as the members of the European Taskforce on Plasma Wall Interaction for supplying their most recent data and publications.

\section{REFERENCES}

1. R. Nygren, et al., Fus. Eng. Design 72, 181-193 (2004).

2. R. J. Colchin, R. E. Clausing, L. C. Emerson, L. Heatherly, and R. C. Isler, "Surface and impurity studies in ORMAK and ISX," in Intern. Symp. on Plasma Wall Interaction, Jülich, (Germany), 18 Oct. 1976, 1976.

3. S. Suckewer, and R. J. Hawryluk, Phys. Rev. Letters 40, 1649-1651 (1978).

4. T. Tanabe, N. Noda, and H. Nakamura, J. Nucl. Mater. 196-198, 11-27 (1992).

5. N. Noda, V. Philipps, and R. Neu, J. Nucl. Mater. 241-243, 227-243 (1997).

6. R. Neu, Physica Scripta T123, 33-44 (2006).

7. S. Itoh, et al., "Ultra-Long Tokamak Discharge by Lower Hybrid Current Drive on TRIAM-1M," in Plasma Physics and Controlled Nuclear Fusion Research 1996, IAEA, Vienna, 1997, vol. 3, pp. 
351-357.

8. M. Apicella, et al., Nucl. Fusion 37, 381-396 (1997).

9. T. Nakano, N. Asakura, H. Kubo, J. Yanagibayashi and Y. Ueda Nucl. Fusion 49, 115024 (10pp) (2009).

10. V. Philipps, et al., Experiments with molybdenum and tungsten limiters in TEXTOR (1994), IAEA, Sevilla, 1994, IAEA-CN-60/A2/A4-P19.

11. A. Pospieszczyk, et al., J. Nucl. Mater. 290-293, 947 - 952 (2001).

12. G. Sergienko, et al., J. Nucl. Mater. 363 - 365, 96 - 100 (2007).

13. K. Behringer, H. Summers, B. Denne, M. Forrest, and M. Stamp, Plasma Phys. Control. Fusion 31, 2059 - 2099 (1989).

14. A. Pospieszczyk, Physica Scripta T119, 71 - 82 (2005).

15. A. Thoma, et al., Plasma Phys. Controlled Fusion 39, 1487-1499 (1997).

16. A. Geier, H. Maier, R. Neu, K. Krieger, and ASDEX Upgrade Team, Plasma Phys. Controlled Fusion 44, 2091-2100 (2002).

17. I. Beigmann, A. Pospieszczyk, G. Sergienko, I. Y. Tolstikhina, and L. Vainshtein, Plasma Phys. Control. Fusion 49, 1833-1847 (2007).

18. K. Asmussen, et al., Nucl. Fusion 38, 967-986 (1998).

19. T. Pütterich, et al., Plasma Phys. Controlled Fusion 50, 085016 (2008).

20. R. Radtke, C. Biedermann, J.-L. Schwob, P. Mandelbaum, and R. Doron, Phys. Rev. A 64, 012720 (2001).

21. S. B. Utter, P. Beiersdorfer, and E. Träbert, Can. J. Phys. 80, 1503-1515 (2002).

22. T. Pütterich, R. Neu, C. Biedermann, R. Radtke, and ASDEX Upgrade Team, J. Phys. B: At. Mol. Opt. Phys. 38, 3071-3082 (2005).

23. Y. Ralchenko, et al., J. Phys. B 41, 021003 (2008).

24. U. Feldman, J. Seely, E. Landi, and Y. Ralchenko, Nucl. Fusion 48, 045004 (2008).

25. C. Biedermann, R. Radtke, R. Seidel, and T. Pütterich, Physica Scripta T134, 014026 (2009).

26. M. Mayer, et al., J. Nucl. Mater. 390-391, 538-543 (2009).

27. K. Sugiyama, et al., "Deuterium inventory in the full tungsten divertor of ASDEX Upgrade," accept. for publ. in Nucl. Fusion (2010).

28. V. Rohde, et al., Nucl. Fusion 49, 085031 (2009).

29. A. Golubeva, V. Kurnaev, M. Mayer, and J. Roth, "Hydrogen retention in plasma-sprayed tungsten," in In Hydrogen in Matter: A Collection from the Papers pres. at the 2nd Int. Symp. on Hydrogen in Matter, AIP Conf. Proc., American Insitute of Physics, 2006, vol. 837.

30. O. Ogorodnikova, J. Roth, and M. Mayer, J. Nucl. Mater. 313-316, 469-477 (2003).

31. O. Ogorodnikova, J. Roth, and M. Mayer, J. Appl. Phys. 103, 034902 (2008).

32. B. Lipschultz, D. Whyte, J. Irby, B. LaBombard, , and G. Wright, Nucl. Fusion 49, 045009 (18pp) (2009).

33. G. Wright, D. Whyte, and B. Lipschultz, J. Nucl. Mater. 390-391, 544-549 (2009).

34. A. Haasz, M. Poon, and J. Davis, J. Nucl. Mater. 266-269, 520-525 (1999).

35. W. Wang, J. Roth, S. Lindig, and C. Wu, J. Nucl. Mater. 299, 124-131 (2001).

36. W. Shu, et al., J. Nucl. Mater. 390-391, 1017 - 1021 (2009).

37. M. Miyamoto, et al., Nucl. Fusion 49, 065035 (2009).

38. S. Takamura, N. Ohno, D. Nishijima, and S. Kajita, Plasma and Fusion Research 1, 051 (2006).

39. M. Baldwin, and R. Doerner, Nucl. Fusion 48, 035001 (2008).

40. S. Kajita, W. Sakaguchi, N. Ohno, N. Yoshida, and T. Sae, Nucl. Fusion 49, 095005 (6pp) (2009).

41. R. Doerner, M. Baldwin, and R. Causey, J. Nucl. Mater. 342, 63 - 67 (2005).

42. M. Baldwin, et al., J. Nucl. Mater. 363-365, 1179 - 1183 (2007).

43. C. Linsmeier, et al., J. Nucl. Mater. 363-365, 1129 - 1137 (2007).

44. K. Schmid, Nucl. Fusion 48, 105004 (2008).

45. W. Eckstein, C. García-Rosales, J. Roth, and W. Ottenberger, Sputtering Data, Rep. IPP 8/82, MPI für Plasmaphysik, Garching (1993).

46. D. Pappas, B. Lipschultz, B. LaBombard, M. May, and C. Pitcher, J. Nucl. Mater. 266-269, 635 641 (1999).

47. A. Kallenbach, et al., Nucl. Fusion 49, 045007 (2009).

48. O. Gruber, et al., Nucl. Fusion 49, 115014 (2009).

49. W. Eckstein, C. García-Rosales, J. Roth, and J. Lázló, Nucl. Instr. Meth. B83, 95 (1993). 
50. D. Naujoks, et al., Nucl. Fusion 36, 671-687 (1996).

51. R. Dux, et al., J. Nucl. Mater. 390-391, 858-863 (2009).

52. B. Lipschultz, et al., Nucl. Fusion 41, 585-596 (2001).

53. B. Lipschultz, et al., J. Nucl. Mater. 363-365, 1246-1250 (2007).

54. R. Dux, et al., J. Nucl. Mater. 363-365, 112-116 (2007).

55. R. Neu, et al., Physica Scripta T138, 014038 (2009).

56. R. Dux, et al., Plasma Phys. Controlled Fusion 45, 1815-182.

57. A. Herrmann, et al., J. Nucl. Mater. 390-391, 747-750 (2009).

58. V. Rohde, M. Balden, T. Lunt, and ASDEX Upgrade Team, Physica Scripta T138, 014024 (2009).

59. G. Sergienko, et al., Physica Scripta T128, 81 - 86 (2007).

60. R. Dux, et al., J. Nucl. Mater. 313-316, 1150-1155.

61. T. Pütterich, E. Wolfrum, R. Dux, C. F. Maggi, and ASDEX Upgrade Team, Phys. Rev. Let. 102, 025001 (2009).

62. R. Dux, T. Pütterich, A. Janzer, and ASDEX Upgrade Team, "Flushing and Erosion of Tungsten during Edge Localized Modes," in Europhysics Conference Abstracts (CD-ROM, Proc. of the 36th EPS Conference on Plasma Physics, Sofia, 2009), ed. by M. Mateev and E. Benova, EPS, Geneva, 2009, vol. 33E, p. O5.056.

63. P. T. Lang, et al., Nucl. Fusion 45, 502-511 (2005).

64. A. Kallenbach, et al., Plasma Phys. Controlled Fusion 47, B207-B222 (2005).

65. R. Neu, et al., Nucl. Fusion 45, 209-218 (2005).

66. C. Angioni, R. Dux, E. Fable, A. G. Peeters, and ASDEX Upgrade Team, Plasma Phys. Controlled Fusion 49, 2027-2043 (2007).

67. J. Rapp, et al., Plasma Phys. Control. Fusion 39, 1615-1634 (1997).

68. R. Dux, C. Giroud, K.-D. Zastrow, and contributors to the EFDA-JET work programme, Nucl. Fusion 44, 260-264 (2004).

69. D. Pacella, et al., Phys. Rev. E 61, 5701-5709 (2000).

70. J. E. Rice, et al., Nucl. Fusion 42, 510-519 (2002).

71. R. Koch, et al., Fus. Eng. Design 26, 103 (1995).

72. R. Neu, et al., Plasma Phys. Controlled Fusion 44, 811-826 (2002).

73. W. West, et al., Physics of Plasmas 9, 1970-1981 (2002).

74. R. Pitts, et al., "Issues associated with the use of W in ITER," in 12th Europ. Fus. Physics Workshop (EFPW), Dec. 7-9, 2009, Velence, Hungary, 2009.

75. A. Loarte, et al., Nucl. Fusion 47, S203-S263 (2007).

76. A. Loarte, et al., Physica Scripta T128, 222-228 (2007).

77. A. Hassanein, T. Sizyuk, and I. Konkashbaev, Fus. Eng. Design 60, 527-546 (2002).

78. B. Bazylev, et al., J. Nucl. Mater. 390-391, 810-813 (2009).

79. J. Roth, et al., J. Nucl. Mater. 390-391, 1-9 (2009).

80. J. Roth, et al., Plasma Phys. Control. Fusion 50, 103001 (2008).

81. W. Wampler, and R. Doerner, Physica Scripta T138, 014037 (2009).

82. M. Baldwin, R. Doerner, D. Nishijima, K. Tokunaga, and Y. Ueda, J. Nucl. Mater. 390-391, 886-890 (2009).

83. K. Schmid, K. Krieger, A. Kukushkin, and A. Loarte, J. Nucl. Mater. 363-365, 674-679 (2007).

84. T. Evans, et al., Nucl. Fusion 45, 595-607 (2005).

85. T. Evans, et al., Nucl. Fusion 48, 024002 (2008).

86. Y. Liang, et al., Phys. Rev. Letters 98, 265004 (2007).

87. A. Alfier, et al., Nucl. Fusion 48, 115006 (2008).

88. M. Fenstermacher, et al., J. Nucl. Mater. 363-365, 476-483 (2007).

89. G. N. Pereverzev, C. Angioni, A. G. Peeters, and O. V. Zolotukhin, Nucl. Fusion 45, 221-225 (2005).

90. R. Dux, et al., "Impurity Transport and Control in ASDEX Upgrade," in Proc. of the 20th IAEA Conference Fusion Energy (CD-Rom), Vilamoura, Portugal, November 2004, IAEA, Vienna, 2005, vol. IAEA-CSP-25/CD, pp. IAEA-CN-116/EX/P6-14.

91. C. Angioni, and A. G. Peeters, Phys. Rev. Let. 96, 095003-1 (2006).

92. H. Bolt, et al., J. Nucl. Mater. 307-311, 43-52 (2002).

93. R. Raffray, et al., Fus. Engin. Design 85, 93 (2009).

94. G. Matthews, et al., Physica Scripta T128, 137-143 (2007).

95. J. Brooks, et al., Nucl. Fusion 49, 035007(6pp) (2009). 